\author{
Proceedings of the $9^{\text {th }}$ International Conference on Applied Informatics \\ Eger, Hungary, January 29-February 1, 2014. Vol.1. pp.9-18 \\ doi: 10.14794/ICAI.9.2014.1.9
}

\title{
Solving algebraic equations with Fibonacci sequences
}

\author{
Gunter Weiss \\ University of Technology Vienna \\ weissgunter@hotmail.com
}

\begin{abstract}
It is well known that the classical Fibonacci sequence $0,1,1,2,3,5,8, \ldots$, $F_{n}, \ldots$ can be used to define the Golden Mean $\phi$ as the $\operatorname{limit}_{n \rightarrow \infty} \frac{F_{n+1}}{F_{n}}$. This limit value is the (positive) solution of the quadratic equation $x^{2}-$ $x-1=0$. To visualize the Fibonacci sequence one uses a nested set of squares and derives a construction of "the" Golden Spiral, a bi-arc spiral consisting of quarter circles. The mentioned procedures easily can be applied to algebraic equations of higher order. To each such an equation it is possible to find a Fibonacci type sequence such that one real solution (if existing) becomes the limit of the quotient of consecutive elements of that sequence. In case of an existing limit value one has therewith an additional way to find solutions of algebraic equations, besides Newton's method and the regula falsi. Mathematics and Informatics courses for high schools mostly deal with algebraic problems, therefore the presented method seems to be well-suited to be introduced into such courses. The paper also reflects the problem of mathematical visualisation. The existing visualisations of Fibonacci sequels are rather standardised and the paper also tries to open up for other and perhaps more adequate visualisations.
\end{abstract}

Keywords: Fibonacci sequel, Golden Mean, elementary algebraic equations

MSC: 26A09, 40A05, 51F05, 51M05

\section{Some facts about the Golden Mean}

The "Golden Mean" (GM) or "Golden Section" and its properties are so well-known that one hardly needs to recall some facts about it. This concept connects Mathematics, Geometry, Biology and, last but not least, Fine Art, see e.g. [3, 11, 12, 15]. While for Mathematics the GM means an interesting number $\phi=1.618 \ldots$, it means a special proportion for Geometry and Art, see Figure 1.

The segment $M$ called the "maior" is the geometric mean of the two segments $m$, the "minor" and the sum $M+m$ and we receive $\phi$ as the ratio $|M|:|m|$ of lengths 


\section{$\begin{array}{lllll}A & M & C & m & B\end{array}$}

Figure 1: "Golden" Proportions of three segments $M, m$ and $M+m$ :

$$
m: M=M:(M+m)
$$

of these segments. The collinear points $A, B, C$ in figure 1 define a the "Golden Ratio" GR, which is the signed value $-\phi^{-1}=1-\phi=-0.618 \ldots$ and both values, $\phi$ and $-\phi^{-1}$, are the two solutions of the quadratic equation

$$
\phi^{2}+\phi-1=0
$$

From the 'ancient Greek point of view' following the Pythagorean commensurability test by dividing the unit segment 1 by $|M|$ receiving $|m|$ as the remainder and then dividing $|M|$ by $|m|$ and so on one gets the GM-value as the result of the continuous fraction

$$
\frac{1}{1+\frac{1}{1+\frac{1}{1+\ldots}}}=0.618 \ldots,
$$

what shows that $\phi$ can be called the "most irrational number".

Remark 1.1. By the way, if we add the ideal point $U$ of the line $A B$ of figure 1 , the four points $A, B, C, U$ form a "Golden Cross Ratio". In general four collinear points define six cross ratio values

$$
x, \frac{1}{x}, 1-x, \frac{1}{1-x}, 1-\frac{1}{x}, \frac{x}{x-1},
$$

but for harmonic point quadruplets there are only three different cross ratio values. For "Golden Quadruplets" there are five, (if we take the absolute values), and these two cases are the only real quadruplets of points with less than six cross ratio values. So there is a projective geometric characterization of the Golden Cross Ratio, too, see [18].

It is also well-known that the GM $\phi$ is the limit of the quotient of consecutive Fibonacci numbers $F_{i}, F_{i+1}$, which are defined recursively by $F_{i+1}=F_{i}+F_{i-1}$. Starting with 0,1 (or more common with 1,1 ) we get the well-known sequel of Fibonacci numbers $0,1,1,2,3,5,8,13,21, \ldots$ but independently of any two real start values the limit of the quotient of consecutive numbers of the resulting sequence always is

$$
\lim _{i \rightarrow \infty} \frac{F_{i+1}}{F_{i}}=\phi .
$$

Traditionally visualization of the Golden Mean happens by "Golden Rectangles", which have side ratio $1:|M|$ (and $|M|: 1$ ) and which om one hand are the limit figures of consecutive Fibonacci squares and on the other have a square as the gnomon figure, see Figure 2. Obviously, by using quarter-circles inscribed to the gnomon squares one receives a discrete logarithmic spiral, which is the result of applying a similarity group to one of the quarter-circles. From the $C A G D$ point of view this spiral is a $G C^{1}$ - circular bi-arc spiral. 

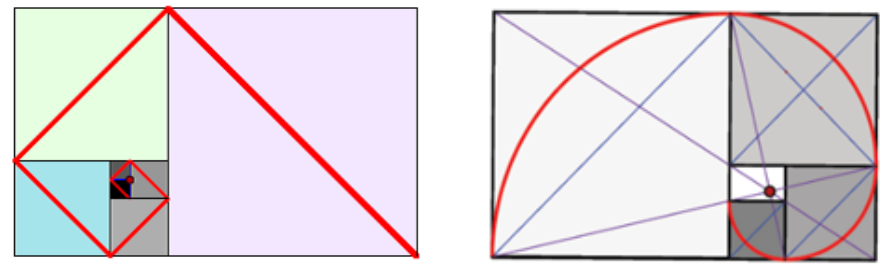

Figure 2: Consecutive Fibonacci squares and "Golden rectangles" with quarter-circle spiral

Of course there are many other visualizations of spiral polygons and circular bi-arc spirals possible which, with the same justification, can be called "Golden Spirals", too, see Figure 3.
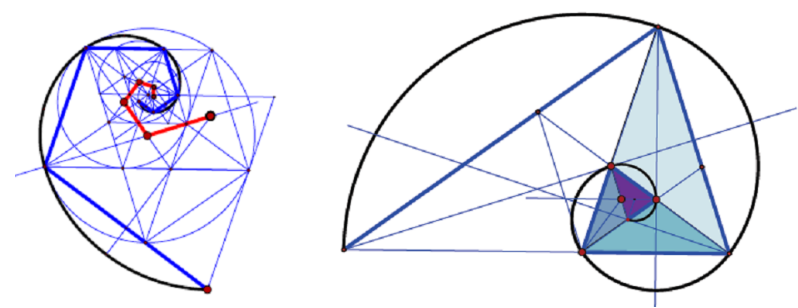

Figure 3: "Golden circular bi-arc spirals derived from the regular pentagon. Left: $72^{\circ}$-circular arcs. Right: $108^{\circ}$-circular arcs.

\section{Solving algebraic equations via Fibonacci sequences}

\subsection{Quadratic equations}

In 1 . we have noticed that $x^{2}-x-1=0$, resp. $x^{2}+x-1=0$ have $\phi$ resp. $1 / \phi$ as positive solutions. Here belong the Fibonacci sequel and thus the limit of "Fibonacci quotients" give the (positive) solution of these special quadratic equations. So one might ask, given a general quadratic equation $x^{2}-a x-b=0, a, b \in$ math $b b R$, find a Fibonacci-type sequel such that the positive solution is the limit of the quotient of adjacent elements of this sequel!

Let us start with a Fibonacci-type sequel defined by $F_{i+1}=p F_{i}+q F_{i-1}$, then it follows that

$$
\lim _{n \rightarrow \infty}\left(1=p \frac{F_{i}}{F_{i+1}}+q \frac{F_{i-1}}{F_{i+1}}\right)=\left(1=\frac{p}{x}+\frac{q}{p x+q}\right)=\left(1=\frac{p}{x}+\frac{q}{x^{2}}\right) .
$$


Now, by comparing coefficients we finally get $p:=a, q:=b$. Therewith we can receive one solution of a quadratic equation with two non-trivial coefficients by calculating the limit of "Fibonacci quotients", i.e. the limit of the sequel of ratios of two adjacent Fibonacci numbers. Note that the quotient sequence is convergent, if and only if the quadratic equation has real solutions.

\subsection{General cubic equations}

Now we shall apply the method described in 2.1 to cubic equations: Given a general cubic equation $x^{3}+a x^{2}+b x+c=0, a, b, c \in \mathbb{R} \backslash\{0\}$, find a Fibonacci-type sequel such that a real solution is the limit of the quotient of adjacent elements of this sequel! Again we start with a Fibonacci-type sequel now defined by $F_{i+1}=$ $p F_{i}+q F_{i-1}+r F_{i-2}$. A short calculation shows that

$$
\lim _{n \rightarrow \infty}\left(1=p \frac{F_{i}}{F_{i+1}}+q \frac{F_{i-1}}{F_{i+1}}+r \frac{F_{i-2}}{F_{i+1}}\right)=\cdots=\left(1=\frac{p}{x}+\frac{q}{x^{2}}+\frac{r}{x^{3}}\right) .
$$

Comparing coefficients we receive $p:=-a, q:=-b, r:=-c$. Note that the generalized Fibonacci-quotient sequence is surely convergent, as long as at least $q \neq 0 \wedge r \neq 0$. Cubic equations always possess at least one real solution.

Remark 2.1. For the special cubic equation $x^{3}+c=0$ the sequel defined by

$$
F_{i+1}=0 \cdot F_{i}+0 \cdot F_{i-1}-c \cdot F_{i-2}
$$

is not a proper Fibonacci-type sequel, but only a geometric sequel. One might have the idea to apply the inverse of a Tschirnhaus-Bring-Ferrard-transformation (TBF-trafo), see [21], to transform the special equation to a general one. The well-known TBF-trafo "deletes" the two first coefficients of an algebraic equation. It means that it is possible to transform a polynomial

$$
x^{n}+a_{n-1} x^{n-1}+a_{n-2} x^{n-2}+a_{n-3} x^{n-3}+\cdots+a_{1} x+a_{0}=0, a_{0} \in \mathbb{R},
$$

into one with $a_{n-1}=a_{n-2}=0$. In our special case a re-transformation by e.g. $x=y-1$ or $x=\frac{1-z}{z}$ would work to receive a general cubic equation. This principle is also the mathematical background for the origami operations to fold the "Delian Problem" $\sqrt[3]{2}$. From $z^{3}-2=0$ one gets $3 x^{3}-3 x^{2}+3 x-1=0$ by the transformation $z:=\frac{1-x}{x}$. If we use a square with unit side length, then the paper folding operations shown in Figure 4 lead to the ratio $1: \sqrt[3]{2}$ as the ratio $x:(1-x)$ on one of the square's sides, see e.g. [19].

Note that "doubling the cube" is equivalent to "halving a prism" into two prisms similar to the given one, which is the 3D-analog to W. OSTWALD's DIN-paper format: $a: b=1: \sqrt{2}$. Such a prism would have the edge length ratios $a: b: c=\sqrt[3]{4}: \sqrt[3]{2}: 1$. This idea can easily be generalized to "n-dimensional Ostwald-prisms", which then would have edge length ratios $2^{(n-1) / n}: 2^{(n-2) / n}: \ldots: 2^{1 / n}: 1$, see [19]. 


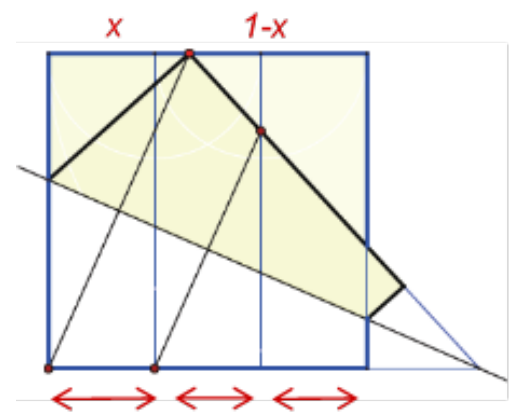

Figure 4: Origami solution of the Delian Problem of doubling the cube

\subsection{The cubic equations of van der LAAN and ROSENBUSCH}

There are and were many attempts to generalize the Golden Mean:

V. SPINADEL e.g. generalizes the defining "Golden" quadratic equation(s) $x^{2}-$ $x-1=0$ resp. $x^{2}+x-1=0$ to $x^{2}+p x-1=0$ and $x^{2}+p x-q=0, p, q \in \mathbb{Z}$, which have the so-called (generalized) "Metallic Means" MM as positive solutions, see [10, 11]. Even it does not really make sense they were thought to be connected to two dimensional visualizations, thus stimulating the question for analogues of the GM and the MMs in three dimensions and connect such mean values with a cubic equations.

It was first Hans van der Laan, a Dutch monk and architect, who proposed to use the equation

$$
x^{3}-x-1=0
$$

and its real solution $\psi=1,324717968 \cdots \approx \frac{4}{3}$, which he called the "Plastic Number" for such a "natural 3D-generalization" of the GM. He used the Plastic Number and its powers as ratios to design proportions of houses, rooms and furniture, c.f. $[1,2,5,7,13]$.

The German Architect Lambert Rosenbusch independently used the equation

$$
x^{3}+x-1=0
$$

and called its real solution $\varrho=0,6823278040 \cdots \approx \frac{2}{3}$ the "Cubi Ratio", c.f. [9, 19]. He also gave a simple planar construction of the Cubi Ratio based on the intersection of a circle and a parabola. He even used the emerging figure as his logo, see Figure 5.

We might ask now for a Fibonacci-type sequel to those special numbers $\psi, \varrho$ : From the defining equations and according to 2.2 we get $p:=0, q:=1, r:=1$ for the sequel defining van der Laan's "Plastic Number" $\psi$ as ratio limit of adjacent 


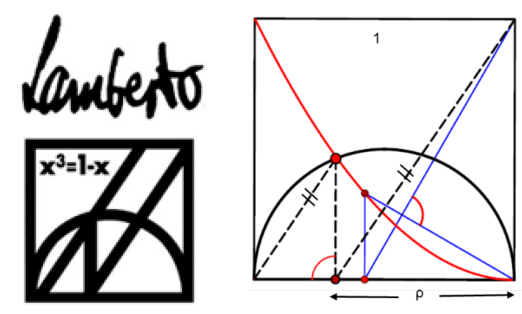

Figure 5: Logo of the architect Lambert Rosenbusch and construction of the Cubi Ratio

numbers of the sequel. Using $1,1,1$ as the first three start numbers we receive the so-called "Padovan sequel" $1,1,1,2,2,3,4,5,7,9,12, \ldots$, see [7]. Similarly, with $p:=0, q:=-1, r:=1$ we get Rosenberg's "Cubi Ratio" $\varrho$ as ratio limit of adjacent numbers of the emerging oscillating sequel $-1,1,1,-2,0,3,-2,-3,5,1,-8,4,9, \ldots$.

\subsection{General algebraic equations}

To find a (real) solution of an algebraic equation

$$
x^{n}+a_{1} x^{n-1}+a_{2} x^{n-2}+\cdots++a_{n-1} x+a_{n}=0, \quad a_{i} \in \mathbb{R},
$$

we look for the corresponding Fibonacci-type sequel putting

$$
F_{i+1}:=p_{1} F_{i}+p_{2} F_{i-1}+\cdots+p_{n} F_{i-n+1} .
$$

Then

$$
\lim _{i \rightarrow \infty}\left(1=p \frac{F_{i}}{F_{i+1}}+p_{2} \frac{F_{i-1}}{F_{i+1}}+\cdots+p_{n} \frac{F_{i-n}}{F_{i+1}}\right)=\cdots=\left(1=\frac{p_{1}}{x}+\frac{p_{2}}{x^{2}}+\cdots+\frac{p_{n}}{x^{n}}\right)
$$

and by comparing coefficients follows $p_{j}=-a_{j}, j=1, \ldots, n$.

Remark 2.2. Note that we only can calculate a real solution of the given algebraic equation as limit of "generalized Fibonacci number quotients", if the sequence of these quotients is convergent. Here one had to apply standard convergence criteria at first. One can freely choose the start values for the generalized Fibonacci number sequence, because the limit of the sequel of quotients does not depend on the start values. Should the given equation only have one nontrivial coefficient, one again will have to apply the invers of a TBF-transformation to get a more general equation. Furthermore, there are no criteria for irreducibility of the given equation and, finally, it is not possible to get (pairs of) complex solutions. But Regula Falsi and Newton's method have the same disadvantages. Their advantage is their applicability to any (smooth differentiable) function. Even so the proposed third method might be of interest for (high) school mathematics resp. informatics courses. It involves applications of material one has to teach anyway. 


\section{Remarks concerning visualization of Fibonacci numbers}

Generalized Fibonacci numbers and solutions of equations are just numbers and thus dimensionless. Usual visualization happens in the plane of nad've perception understood as Euclidean plane. As Geometry has proportions instead of 0 and 1 one has to endow the plane with an arbitrarily posed Cartesian frame and an arbitrarily chosen unit.

Standard visualization uses squares and a $C^{1}$-biarc spline of quarter circles, see Figure 6 .
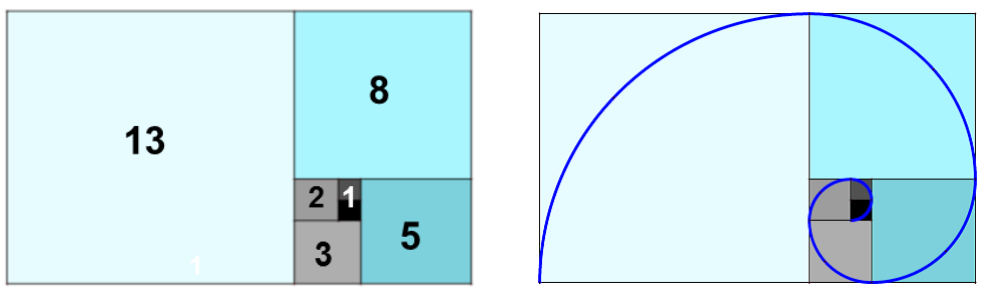

Figure 6: Fibonacci squares and quarter circle Fibonacci-spiral

But the nad've place of action also could be considered to be e.g. a hyperbolic world, where we do not have squares. But circles exist as well in the Euclidean plane as in the hyperbolic plane and one could replace the squares by touching circles of radii $r_{i+1}=r_{i}+r_{i-1}$ according to the Fibonacci numbers, see Figure 7.

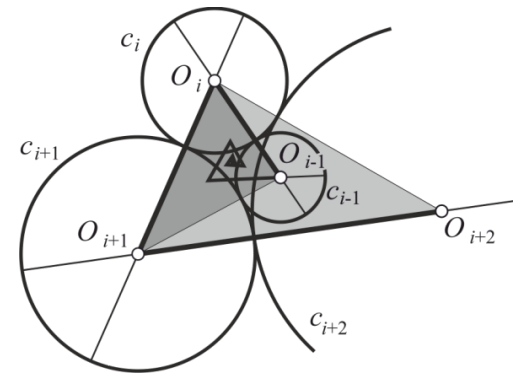

Figure 7: Chain of touching "Fibonacci circles"

Here we consider only the Euclidean situation. While the convex hull of the squares always is a rectangle, the side ratio of which tends to that of a "Golden Rectangle" in the limit, the convex hull of the centers $O_{i}$ of the Fibonacci circles is a triangle with a side ratio

$$
\left(r_{i-1}+r_{i}\right):\left(r_{i-1}+r_{i+1}\right):\left(r_{i-1}+r_{i+1}\right)
$$


with the ratio $\phi^{2}: \phi^{3}:\left(1+\phi^{2}\right)$ in the liomit. A triangle with such a side ratio can be called a "Golden triangle" (in spite of that this concept is already used for an isosceles triangle with side ratio $1: \phi: \phi)$.

Remark 3.1. "Visualization" means "mapping" (of mathematical facts) plus "interpretation" (by a viewer, another person). As visualization criteria one finds: Simplicity, unique interpretation, aesthetics. Hidden assumptions are the cultural and educational background of the viewer (and of the mapping person). Obviously this educational background is the reason for preferring Euclidean geometry as standard place of action. But sometimes Euclidean Geometry is not the most clever place of action for visualizing a special mathematical fact. E.g. hyperbolic geometry, space-time geometry, the geometry of (general) metric spaces, Möbius Geometry and so on are sometimes logically better suited to visualize a special mathematical idea. Euclidean visualizations often "hide", where a mathematical fact really belongs. As an example we present George Odom's discovery of the Golden Mean occurring in a figure consisting of an equilateral triangle and its circumcircle, see [4], [20] and Figure 8 (left). Historically the Golden Mean is a ratio of segments and "ratio" is an affine geometric concept. Therefore any affine transform of Odom's figure will show the Golden Mean, too, see Figure 8 (right).
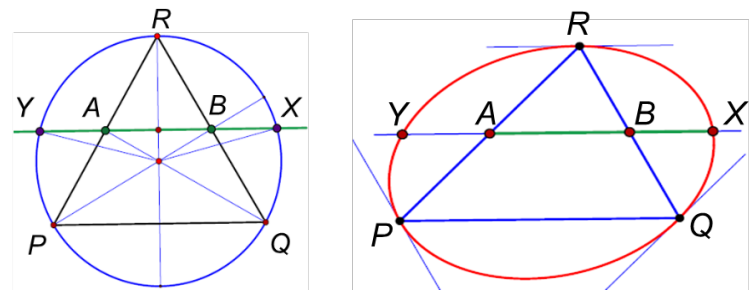

Figure 8: G. Odom's construction of the Golden Mean (left) and affine transform (right)

The spiral arrangement of Golden Rectangles with squares as gnomons depends only on two orthogonal directions in the Euclidean plane. An affine transformation of this arrangement allows to state that, with respect to a suitable affine coordinate frame, any parallelogram can be called "Golden Parallelogram", c.f. [6].

For the definition of a $G C^{1}$-bi-arc spiral consisting of quarter circles we only need a general metric space, i.e. a Minkowski plane. In such a plane translations and centric similarities (dilatations) exist, but there are in general no rotations possible. Also in such a general Minkowski plane it is possible to construct analogues of Fibonacci quarter circle spirals and logarithmic biarc spirals using centric similarities and translations alone, see e.g. Figure 9.

We end this chapter with generalizing the classical Golden Rectangle and the golden quarter circle spiral to Golden Prisms and spirals in higher dimensional Euclidean spaces, still having in mind that $\phi$ is a dimensionless number and the classical visualization happens by a ratio of three collinear points defining segments, which are one-dimensional objects. Figure 2 (right) shows the two-dimensional 


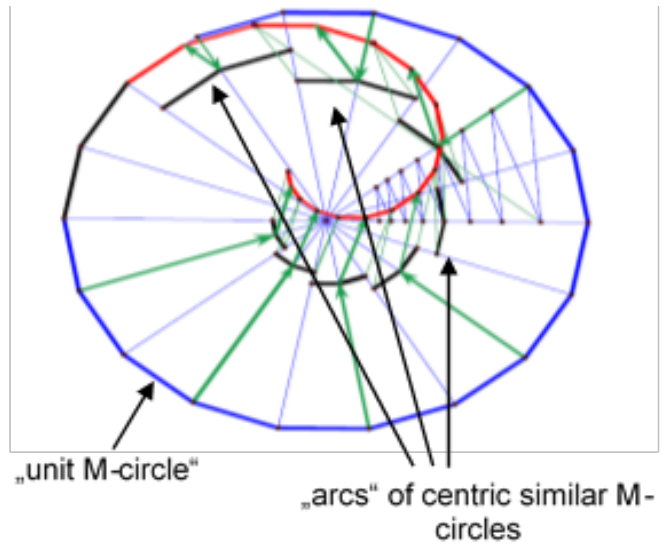

Figure 9: Minkowski plane with a centric symmetric polygon as unit circle and construction of a "spiral" with Minkowski-circular $\operatorname{arcs}$

visualization by a nersted set of Golden Rectangles with squares as gnomons. A three-dimensional generalization could take e.g. prisms with side ratio 1: $\phi: \phi^{2}$, from which one can cuts off "gnomon prisms" with side ratio 1:1: $\phi$. But one could also start with cubes with sides $1, a, a^{2}, a^{3}, a^{4}, \ldots$ in a spiral arrangement. If $a$ fulfills the condition $a=\phi-1$ we get a nested set of "Golden Prisms" with side length ratio 1:1: $\phi$ and can replace the spiral polygon formed by the cubes' diagonals by helical arcs or by Bézier splines, see [17] and Figure 10.

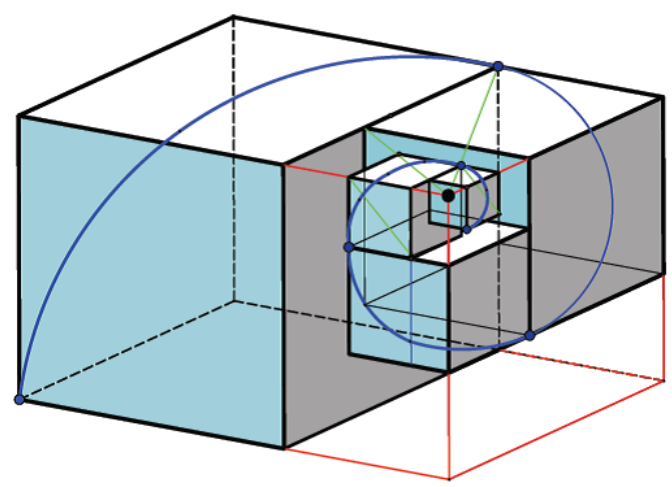

Figure 10: Nested set of "Golden Prisms" with Golden Helical spiral

It is obvious, how to proceed in higher dimensions. Also for other "closure conditions" which generalize the "golden condition" $\phi^{2}-\phi=1$ to those of Metallic Means or the Plastic Number and the Cubi Ratio one can find similar visualizations based on "Metallic Prisms", "Laan Prisms" and "Rosenbusch Prisms", see [17]. 


\section{References}

[1] AARTS, J., FOKKINK, R., KRUiJTZER, G., Morphic Numbers, NAW 5/2 Nr.1 (2001), p.56-58.

[2] ALSINA,C., J.L. GARCI-ROIG, J.L., On plastic numbers, Journ. Math.\& Design, Vol.1 No.1 (2001), p.13-19, ISSN 1515-7881

[3] A. Beutelspacher, A., PETRI, B., Der Goldene Schnitt, $2^{\text {nd }}$ ed., SpektrumAkademischer Verlag 1997

[4] COXETER, H.S.M.: Problem E3007, American Mathematical Monthly 1983.

[5] H. v.d. LAAN, H.v.d., Le Nombre Plastic - quinze leçons sur l'ordonnance architectonique, Brill, Leiden 1960.

[6] H. MARTINI, H., SPIROVA, M., Golden rectrangles in normed planes. Mitteilungen. d. Math. Ges. Hamburg 29 (2010), p.125-134 (MR 2012 a: 51019)

[7] PADOVAn, R., Dom Hans van der Laan and the Plastic Number. Nexus Network Journal Vol.IV(3), Architecture and Mathematics (2002), p.181-193 [pdf].

[8] REDONDO-BUITRAGO, A., Sobre los sistemas de proporciones áureo y plastic y sus generalizaciones. Journ. Math. \& Design, Vol. 9(1) (2009), p.15-34. ISSN 1515.7881

[9] ROSENBUSCH, L., Räumliche Proportionen. (In: Geometrie, Kunst und Wissenschaft 06, ed. O. Niewiadomski), Hauschild Verlag 2007, ISBN 978-38975-366-6.

[10] SPINADEL, V. W. de, The family of Metallic Means. Visual Mathematics 1(3) (1999), http://members.tripod.com/vismath1/spinadel.

[11] SPINADEL, V. W. de, Golden and Metallic Means in Modern Mathematics and Physics. Proc. $13^{\text {th }}$ ICGG 2008, Dresden, Germany, ISBN 978-3-86780-042-6.

[12] SPINADEL, V. W. de, From the Golden Mean to Chaos. $3^{\text {rd }}$ ed. 2010, Nueva Librería, Buenos Aires, Argentina, ISBN 978-987-1104-83-3.

[13] SPINADEL, V. W. de, REDONDO-BUITRAGO, A., Towards van der Laan's Plastic Number in the plane. JGG Vol. 13(2), (2008), p.163-175.

[14] SPINADEL, V. W. de, REDONDO-BUITRAGO, A., Generalizing the Golden Spiral. Journ. Math.\& Design Vol. 11(1) 2011, ISSN 1515.7881.

[15] STAKHOV, A., Mathematics of Harmony. From Euclid to Contemporary Mathematics and Computer Science. Series on Knots and Everything, Vol.22 (1998) and World Scientific Publ. Co., London (2008).

[16] THOMPSON, A.C., Minkowski Geometry. Cambridge Univ. Press 1996, ISBN 0521-40472-X

[17] G. WEISS, G., From the Golden Rectangle to the Laan Box and more. (occurs in Journ. Math.\& Design Vol. 12(1) 2012).

[18] G. WEISS, G., Golden Hexagons - Computer Generated Figures with Remarkable Properties, Proceedings ICAI99, Eger-Noszvaj, HU, p.9-21.

[19] G. WEISS, G., SPINADEL, V. W., Remarks to classical cubic problems and the mean values of van der Laan and Rosenbusch. Proc. $14^{\text {th }}$ ICGG 2010, Kyoto, Japan, Art. 235, p. 1-11. ISBN 987-4-9900967-1-7

[20] G. WEISS, G., SPINADEL, V. W., From George Odom to a new system of Metallic Means, (occurs in Journ. Math.\& Design Vol. 13(1) 2013).

[21] WIKIPEDIA, http://de.wikipedia.org/wiki/Tschirnhaus-Transformation. 